\title{
EXISTE FUNDAMENTO CIENTÍFICO PARA O TRATAMENTO DA FRATURA DE FÊMUR PELA HIDROTERAPIA? UMA REVISÃO INTEGRATIVA
}

\author{
IS THERE SCIENTIFIC BASIS FOR THE TREATMENT OF FEMORAL FRACTURE \\ BY HYDROTHERAPY? AN INTEGRATIVE REVIEW
}

\section{Francisco Eudison da Silva Maia ${ }^{a}$, Fabio Firmino de Albuquerque Gurgel ${ }^{b}$}

aeudisonmaia@yahoo.com.br, bwell-felipe@hotmail.com

Universidade Potiguar - Mossoró (RN), Brasil

Data de recebimento do artigo: 28/09/2015

Data de aceite do artigo: 25/04/2016

\section{RESUMO}

Introdução: A fratura do fêmur é complexa, devido à gravidade, ao tempo de reabilitação e às repercussôes individuais e socioeconômicas. Nesta perspectiva, a hidroterapia é um método de tratamento que visa a maior eficiência e ganho funcional em menor tempo. Objetivo: Este artigo tem como intuito revisar criteriosamente se há respaldo na literatura para o uso da hidroterapia nesse tipo de patologia. Metodologia: Este trabalho caracteriza-se como uma revisão de literatura, a partir de publicaçôes no período de 2000 a 2014, adotando como critérios de inclusão estudos nos idiomas português, inglês e espanhol, sem distinçáo de gênero e idade e com o uso de descritores predeterminados. Resultados: A literatura atual não registra resultados que comprovem a eficácia da hidroterapia para pacientes com esse tipo de fratura. Conclusóes: A hidroterapia ainda se fundamenta em conhecimento empírico e vivências clínicas, o que denota uma maior necessidade de pesquisas acerca deste assunto.

Palavras-chave: Fraturas do fêmur; hidroterapia; reabilitação.

\section{ABSTRACT}

Introduction: A femoral fracture is complex due to its severity, rehabilitation time, and individual and socioeconomic repercussions. In this perspective, hydrotherapy is a treatment method that seeks better efficiency and functional gains in a shorter time. Objective: The aim of this article is to review carefully whether there is support in the literature for the use of hydrotherapy in this type of pathology. Method: This study is characterized as a literature review, based on publications in the period between 2000 and 2014, accepting as criteria for inclusion studies in Portuguese, English, and Spanish, irrespective of gender and age, using predetermined descriptors. Results: The current literature does not record results demonstrating the efficacy of hydrotherapy for patients with femur fracture. Conclusion: The use of hydrotherapy is still based on empirical knowledge and clinical experiences, which indicates a greater need for research on this subject.

Keywords: Femoral fractures; hydrotherapy; rehabilitation. 


\section{Introdução}

A fratura do fêmur pode causar impactos negativos para o indivíduo em um ambiente socioeconômico em desenvolvimento como o nosso, gerando na maioria dos casos impossibilidade para realizar as atividades de vida diária, limitaçóes funcionais, dor intensa, deformidade, edema, sensibilidade local acentuada ou espasmos musculares ${ }^{1}$. É estimado para este milênio 215 mil fraturas do colo do fêmur por ano na população brasileira ${ }^{2}$.

Diante dessa realidade e pensando em uma reabilitação mais eficiente, com um maior ganho de funcionalidade em um menor tempo possível, a hidroterapia vem sendo indicada e utilizada por médicos e fisioterapeutas em programas multidisciplinares nas mais diversas condiçōes clínicas ${ }^{3}$.

A hidroterapia é um dos recursos mais antigos da fisioterapia, consiste no uso externo da água com propósitos terapêuticos, associando os efeitos físicos, fisiológicos e cinesiológicos advindos da imersão do corpo no meio aquático como recurso para a reabilitaçáo ou na prevenção de alteraçóes funcionais ${ }^{3}$.

A prática da reabilitação no meio aquático está sendo cada vez mais indicada, devido aos diversos benefícios promovidos pela força do empuxo, como a possibilidade de descarga de peso precoce, diminuição do peso corporal e maior liberdade de movimentos ${ }^{4}$.

Não obstante os diversos benefícios provenientes do tratamento usando a hidroterapia, esse método ainda não se configura significativamente como escolha de tratamento para reabilitação de pacientes que sofreram fratura do fêmur ${ }^{5}$. Com o objetivo de discutir se a reabilitação desses pacientes por esse método é um tratamento que tem base científica, realizaremos um levantamento bibliográfico sistemático.

\section{Metodologia}

Este artigo caracteriza-se como uma revisão de literatura tipo integrativa, que visa tratar sobre os fundamentos científicos da hidroterapia como método de reabilitação para paciente com fratura do fêmur.

Foram utilizados como fontes de referência artigos das bases de dados SciELO (Scientific Electronic Library Online), Lilacs (Literatura Latino-Americana e do Caribe em Ciências da Saúde), PubMed (National Library of Medicine e National Institutes of Health), Ibecs (Índice Bibliográfico Espanhol de Ciências da Saúde), MedLine (Medical Literature Analysis and Retrieval System Online), Registro Cochrane Library (Cochrane de Ensaios Clínicos Controlados) e do sistema de busca do Google Acadêmico. A ampliação da busca literária ocorreu visando uma maior abrangência e devido às dificuldades em encontrar estudos específicos. Vale salientar que os estudos que foram utilizados são de caráter multidisciplinar, justificado pelo fato supracitado. Para realizar a busca nas bases de dados foram utilizados os seguintes descritores: "fraturas do fêmur", "hidroterapia", "reabilitação" e "fisioterapia", com seus similares em inglês e espanhol.

A literatura consultada foi publicada no período de 2000 a 2014, e os critérios de inclusão foram estudos nos idiomas português, inglês e espanhol, que registrasse lesão em qualquer região femoral e seu respectivo tratamento, sem distinção de gênero e idade. Para a delimitação dos artigos, foram utilizados aqueles que apresentaram os termos "fraturas do fêmur", "hidroterapia" e "reabilitação" em qualquer um dos campos do título, resumo ou palavras-chave.

Em relação aos critérios de exclusão, os estudos que não apresentaram categoricamente o tipo de fratura e as condutas foram automaticamente excluídos.

Foram utilizados para a elaboração deste trabalho 54 artigos das bases de dados, além de seis livros sobre o tema, totalizando 60 referências ${ }^{1-60}$. A pesquisa foi realizada entre novembro de 2013 e agosto de 2014.

\section{Resultados}

A Tabela 1 mostra as bases de dados utilizadas, o período analisado e a quantidade de publicaçóes utilizadas na pesquisa.

Tabela 1: Bases de dados e publicações encontradas no período de 2000 a 2013.

\begin{tabular}{lcc}
$\begin{array}{l}\text { Base de } \\
\text { dados }\end{array}$ & $\begin{array}{c}\text { Período utilizado para } \\
\text { realizar a busca nas bases } \\
\text { de dados }\end{array}$ & $\begin{array}{c}\text { Quantidade de } \\
\text { publicaçóes afins }\end{array}$ \\
\hline SciELO & $2000-2013$ & 23 \\
\hline Lilacs & $2000-2013$ & 7 \\
PubMed & $2000-2013$ & 3 \\
Ibecs & $2000-2013$ & 1 \\
Medline & $2000-2013$ & 2 \\
\hline $\begin{array}{l}\text { Registro } \\
\text { Cochrane }\end{array}$ & $2000-2013$ & 1 \\
Library & & 17 \\
Google & $2000-2013$ & 54 \\
Acadêmico & & \\
Total & & \\
\hline
\end{tabular}

Fonte: Elaborado pelos autores, 2015.

A Tabela 2 aponta e organiza as informaçôes e a síntese das principais intervenções, resultados e consequências dos principais achados desta revisão. 
Tabela 2: Síntese dos principais achados da revisão.

\begin{tabular}{|c|c|}
\hline Autor(es) e ano de publicaçáo & Intervençáo e resultados/consequências \\
\hline $\begin{array}{l}\text { Mangione, Craik, Tomlinson, Palombar } \\
2005^{7} \text {; }\end{array}$ & \\
\hline $\begin{array}{l}\text { Araújo, Oliveira, Bracco, } 2005^{8} \text {; } \\
\text { Paschoal, Paccola, } 2000^{9} \text {; } \\
\text { Arliani, Astur, Linhares, Balbachevsky, } \\
\text { Fernandes, Reis, } 2011^{10} \text {; }\end{array}$ & $\begin{array}{l}\text { A fratura do fêmur é considerada um dos acometimentos de maior complexidade devido ó } \\
\text { a sua gravidade, tempo de consolidaçáo e reabilitaçáo, sendo uma das maiores responsá- } \\
\text { veis pela morbimortalidade de pacientes com comorbidades e alto risco de complicaçóes } \\
\text { pós-operatórias. }\end{array}$ \\
\hline
\end{tabular}

Bento, Vidmar, Silveira, Wibelinger, 2011 $1^{11}$;

Souza, Pinheiro, Coeli, Junior, Torres, $2007^{12}$

Bento, Vidmar, Silveira, Wibelinger, 2011 ${ }^{11}$; Buckwalter, $2000^{13}$

Rocha, Carvalho, Zanqueta, Sandro, 20014 Moraes, Silva, Ferreira, Ferro, Rocha, Teixeira, $2009^{15}$

Gomes, Garcia, 2006²;

Arliani, Astur, Linhares, Balbachevsky, Fernandes, Reis, $2011^{10}$

Arliani, Astur, Linhares, Balbachevsky, Fernandes, Reis, $2011^{10}$;

Muniz, Arnaut, Yoshida, Trelha, $2007^{16}$

Barbosa, Camargo, Arruda, Israel, 2006 ${ }^{17}$; Avelar, Bastone, Alcântara, Gomes, $2003^{18}$

Gomes, 2006 ${ }^{19}$;

Candeloro, Caromano, $2007^{20}$

Barbosa, Camargo, Arruda, Israel, $2006^{17}$ Farias, $2009^{21}$

Fonte: Elaborado pelos autores, 2015

\section{Discussões}

A hidroterapia, originada dos termos gregos hydro (de hydor, hydatos = água) e therapeia (tratamento), tem apresentado grande prestígio como alternativa de tratamento para pacientes com diversos comprometimen$\operatorname{tos}^{22}$, sendo caracterizada como um dos recursos mais antigos da fisioterapia ${ }^{2}$.
A possibilidade de ocorrer fratura desse osso é alta, sendo a prevalência em torno de três para cada 10 mil habitantes e com crescimento contínuo.

Fratura do fêmur acontece com maior predominância entre a sétima e oitava décadas de vida, sendo que as transtrocanterianas apresentam maior prevalência entre a oitava e nona e as subtrocanterianas, em número bem menor, na terceira e nona décadas. A área de maior demanda mecânica é a peritrocanteriana, que, por isso, seria a de maior número de fraturas em idade mais jovem, quando a atividade física é maior e a possibilidade de trauma também. Provavelmente, estão envolvidos nesse aspecto vários fatores: potência muscular, direção da força lesiva, distribuição espacial das trabéculas ósseas e ainda fatores individuais. A maior incidência de fraturas peritrocanteriana em indivíduos mais jovens ( 20 a 30 anos) e nos mais velhos na regiáo transtrocanterianas (70 a 80 anos) sugere que essas regióes apresentam demanda mecânica bastante diversa entre si, sendo necessário trauma de maior energia nos jovens, enquanto no idoso o trauma ocorre preferentemente nas regióes mais proximais.

Nos Estados Unidos especula-se que haverá mais de 500 mil fraturas de quadril anuais até 2040, e se espera que os custos para o tratamento cheguem a 16 bilhôes de dólares por ano. Entre o período de 1999 a 2002, constatou-se que os estadunidenses gastaram 3 bilhôes de dólares para tratar esse tipo de paciente. Em vários países, inclusive no Brasil, os custos sociais e econômicos com esse tipo de fratura vêm aumentando a cada ano.

A fratura de fêmur acarreta uma perda significativa da capacidade funcional, em geral $30 \%$ dos pacientes acabam falecendo após um ano da lesão, o que faz ser a principal causa de morte por trauma, principalmente em pessoas com mais de 75 anos de idade.

A hidroterapia é um método terapêutico bastante reconhecido no tratamento de doenças ortopédicas e reumáticas que associa os princípios físicos da água (temperatura, pressão hidrostática, flutuação, turbulência, densidade, viscosidade e tensão superficial) aos fisiológicos e cinesiológicos advindos da imersão do corpo no meio líquido. Esse recurso é amplamente utilizado na reabilitação ou prevenção de alteraçôes funcionais, proporcionando uma recuperação sem dor e com diminuição dos espasmos musculares devido à redução do impacto articular.

Uma das peculiaridades desse método que ficou ressaltada nos estudos são os benefícios que as propriedades físicas da água podem promover ao paciente, como a realização de exercícios com menos sobrecarga corporal, aquilatando o funcionamento do sistema termorregulador, venoso, arterial e capilar. Outro beneficio é que o meio aquático gera uma condição de resistência multidimensional contra os movimentos e atenua a sobrecarga sobre as articulações. A hidroterapia baseia-se em um trabalho específico e individual, respeitando as especificidades de cada paciente, visando aumentar a segurança dele em relação à sua patologia. Sabe-se que um trabalho de recuperação cinético funcional pode ser beneficiado no meio líquido, pois diversos exercícios podem ser realizados com mais segurança e sem riscos de quedas ou novas lesôes. 
pressão hidrostática, viscosidade e turbulência, com a cinesioterapia. Esses diferentes princípios proporcionam aos pacientes diversos efeitos fisiológicos que envolvem praticamente todos os sistemas corporais ${ }^{23,24}$.

Mediante esse fato, a reabilitação de pacientes com fratura do fêmur utilizando esse método deve ser investigada, pois as projeçôes brasileiras para esse milênio incluem 4,3 milhôes de casos de osteoporose, com 215 mil fraturas do colo do fêmur por $\mathrm{ano}^{2}$. Para Carregaro e Toledo ${ }^{5}$, os exercícios realizados na água favorecem a reabilitação pois proporcionam menor estresse articular, aumento da circulação e facilidade para se movimentar.

Diversas pesquisas têm demostrado efeitos significantes na redução da dor ao movimento e melhoras na função física, fato este que determinou melhorias na qualidade de vida dos indivíduos que são tratados com esse método ${ }^{5,25}$. Por isso, alguns autores colocam que a hidroterapia se mostra como um recurso terapêutico de resultados satisfatórios, pois aumenta o equilíbrio, promove ganho de força e propriocepção ${ }^{26}$.

Contudo, é necessário deixar claro que o assunto sobre a reabilitaçáo de paciente com fratura do fêmur usando a hidroterapia é um tema pouco tratado na literatura. $\mathrm{Na}$ realidade, em todos os artigos utilizados para realizar esta revisáo, nenhum trata exclusivamente deste assunto.

Lustosa e Bastos ${ }^{27}$, em uma revisão semelhante, tratando sobre fraturas proximais do fêmur em idosos, descrevem que dentre os objetivos e a conduta de tratamento foram preconizados a mobilização precoce, a descarga de peso assim que permitido, os treinos de transferências e o treino funcional para o retorno gradativo às atividades cotidianas, não fazendo alusão em momento algum ao uso da hidroterapia. $\mathrm{O}$ mesmo fato aconteceu no estudo desenvolvido por Mangione, Craik, Tomlinson, Palombaro ${ }^{7}$.

$\mathrm{Na}$ pesquisa realizada por Pesciallo, Pastrián Mana, Barrios e Del Sel ${ }^{28}$, novamente a hidroterapia não é cogitada como opçáo de tratamento, e sim a descarga de peso parcial gradativamente em solo e o incentivo à deambulação. Para esses autores, o procedimento de descarga precoce de peso no membro afetado é uma boa alternativa para pacientes, principalmente idosos com má qualidade óssea. Para Guimarães, Guimarães e Franco ${ }^{29}$, a deambulação precoce em solo apresenta melhor reabilitação funcional do paciente idoso e alto índice de consolidação decorrente da preservação do envelope de partes moles em torno da lesão.

O tratamento realizado no meio aquático proporciona situaçóes de instabilidade, fator que fornece uma gama de informaçóes sensoriais que promovem a melhora do equilíbrio corporal. Porém, o tratamento realizado fora desse meio se aproxima mais das atividades da vida diária do paciente ${ }^{18,30}$.
Barbosa, Camargo, Arruda e Israel ${ }^{17}$ propóem que a reabilitação cinética funcional da marcha pode ser beneficiada no meio líquido. No entanto, eles fazem questáo de deixar claro que mais estudos precisam ser desenvolvidos, devido à escassez de literatura específica sobre avaliação hidroterapêutica, sendo também preciso desenvolver instrumentos de avaliação para alcançar as evidências científicas de modo claro. Essa mesma ideia também é compartilhada por Meereis, Favretto, Souza, Marques, Gonçalves e Mota ${ }^{31}$.

Katrak, O'Connor e Woodgate ${ }^{32}$, em um relato de caso tratando sobre reabilitaçâo após artroplastia total do fêmur, dizem que técnicas de fisioterapia, como exercícios ativos assistidos, exercícios isométricos e hidroterapia são extremamente úteis durante a fase inicial de reabilitação para facilitar um programa de fortalecimento gradual, mas são enfáticos ao colocar que não encontraram literatura específica sobre o tema e que os aspectos literários generalistas que envolvem a reabilitação são bastante breves.

Já para Campion ${ }^{33}$ e Ruoti, Morris e Cole ${ }^{34}$, em seus respectivos livros, o tratamento hidroterápico tem seus benefícios nas estruturas envolvidas na amplitude de movimento da regiáo do quadril e joelhos.

$\mathrm{Na}$ pesquisa avançada na base de dados digitais do Portal de Pesquisa da Biblioteca Virtual em Saúde (BVS) via descritor "hydrotherapy", colocando como filtro o limite em humanos e o tipo de documento (no caso, artigos), deixando os demais campos desmarcados, foram encontrados 254 manuscritos que tinham em seu título o prescrito supracitado, porém, nenhum relacionado à reabilitação usando a hidroterapia em fratura do fêmur. $\mathrm{Na}$ expansão avançada, adicionando como tipo de documentos ensaio clínico controlado, relato de casos, avaliação de tecnologias em saúde, revisôes sistemáticas avaliadas, estudo de casos e controles, guia de prática clínica e avaliaçáo econômica em saúde, colocando como filtro o limite em humanos, deixando os demais campos desmarcados, o sistema disponibilizou 32 artigos, nenhum dos quais, novamente, se utilizou desse método para reabilitação. Esse método de pesquisa foi escolhido por ser rápido e preciso ao processo de busca $^{35,36}$.

Em uma pesquisa similar, Silva, Santiago e Coelho ${ }^{37}$, ao utilizarem "hidroterapia" como descritor para localizar as produçóes científicas nacionais e internacionais, identificaram 11 artigos, dos quais apenas nove mostravam aplicação em seres humanos. Concluíram argumentando que há carência de estudos que relacionem as práticas de cuidar e a hidroterapia, especialmente pesquisas de campo ou de caso controle.

Com base nos resultados desta revisão, pode-se afirmar que mais estudos precisam ser desenvolvidos, por existirem muitas questôes náo elucidadas acerca da real 
eficácia da hidroterapia para a reabilitação de paciente com fratura do fêmur ${ }^{38}$. No entanto, mesmo com a ressalva, foi possível determinar fortes evidências para os efeitos positivos do tratamento usando a hidroterapia na atenuação da dor, no aumento da mobilidade articular, na força muscular, na melhora da funcionalidade, do equilíbrio e no condicionamento físico ${ }^{38-46}$. Contudo, não foi possível afirmar o que ocorre diretamente com os indivíduos que sofreram lesão do fêmur e que foram tratados com a hidroterapia, simplesmente devido à ausência de estudos e pesquisas específicas 5 . Em outras revisōes de literatura tratando da abordagem hidroterapêutica aplicada a diversas limitaçóes funcionais e patológicas, os autores também são unânimes ao afirmar que não são encontradas evidências literárias para apoiar essa conduta ${ }^{47-60}$.

Com isso, pode-se deduzir que pesquisas ou estudos com metodologias científicas e com amostra suficiente utilizando a hidroterapia no tratamento de fraturas do fêmur não foram encontrados. No entanto, empiricamente seus resultados são considerados positivos por vários profissionais.

Para esse tipo de tratamento faz-se necessário realizar estudos epidemiológicos que incluam vigilância, observação, pesquisa analítica e experimental, análise por tempo, local e características dos indivíduos e principalmente condiçóes relacionadas à saúde, como doenças, hábitos de vida e equipamentos avaliativos adequados.

A literatura atual não mostra resultados que comprovem a eficácia do uso da hidroterapia para reabilitação de pacientes que sofreram fraturas de epífises, metáfises e diáfise do fêmur, o que resulta em uma falta de variáveis seguras para um tratamento tão promissor.

Maia et al..$^{60}$, tratando sobre outro tema bastante discutido no meio fisioterapêutico, dizem ser surpreendente que em algumas condutas ou procedimentos o conhecimento esteja baseado puramente em práticas empíricas ou em vivências clínicas. Por fim, este assunto precisa de mais pesquisas.

\section{Considerações finais}

No decorrer da elaboração desta revisão literária, foi possível abordar o tratamento por meio da hidroterapia em pacientes acometidos de fratura do fêmur.

Mediante o que foi desenvolvido, podemos concluir que esse método necessita de maior aparato científico devido ao constante uso como conduta para reabilitação. Entretanto, cabe ressaltar que o nosso intuito não é finalizar este assunto, e sim levantar questionamentos sobre algo que praticamente não tem base científica e ao mesmo tempo é muito praticado no meio fisioterapêutico.
Com isso, podemos concluir que, levando em consideração os artigos que foram revisados, o tratamento para fraturas de epífises, metáfises e diáfise do fêmur usando a hidroterapia até este momento tem efeitos indefinidos. Por fim, a fisioterapia pode evoluir mais nessa área, sendo necessária a realizaçáo de mais pesquisas em caráter experimental, com maior controle sobre as variáveis.

\section{Referências}

1. Mesquita GV, Lima MALT, Santos AMR, Alves ELM, Brito JNPO, Martins MCC. Morbimortalidade em idosos por fratura proximal do fêmur. Texto \& Contexto Enferm. 2009; 18(1):67-73.

2. Gomes MCSM, Garcia RR. Comparação entre o tratamento no solo e na hidroterapia para pacientes com osteoporose: revisão da literatura. Rev Bras Ciên Saúde. 2006;3(7):45-56.

3. Biasoli MC, Machado CMC. Hidroterapia: aplicabilidades clínicas. Rev Bras Med. 2006;63(5):225-37.

4. Alberton CL, Kruel LFM. Influência da imersão nas respostas cardiorrespiratórias em repouso. Rev Bras Med Esporte. 2009;15(3):228-32.

5. Carregaro RL, Toledo AM. Efeitos fisiológicos e evidências científicas da eficácia da fisioterapia aquática. Rev Movimenta. 2008;1(1):23-27.

6. Sociedade Brasileira de Ortopedia e Traumatologia Colégio Brasileiro de Radiologia. Fratura transtrocanteriana. Rev Assoc Med Bras. 2009;55(6):637-40.

7. Mangione KK, Craik RL, Tomlinson SS, Palombaro KM. Can elderly patients who have had a hip fracture perform moderate to high intensity exercise at home? Physical Therapy. 2005;85(8):727-739.

8. Araújo DV, Oliveira JHA, Bracco OL. Custo da fratura osteoporótica de fêmur no sistema suplementar de saúde brasileiro. Arq Bras Endocrinol Metab. 2005;49(6):897-901.

9. Paschoal, FM, Paccola CAJ. Haste bloqueada "Faculdade de Medicina de Ribeirão Preto": experiência clínica no tratamento das fraturas femorais. Acta ortop bras. 2000;8(4):160-77.

10. Arliani GG, Astur DC, Linhares GK, Balbachevsky D, Fernandes HJA, Reis FB. Correlação entre tempo para o tratamento cirúrgico e mortalidade em pacientes idosos com fratura da extremidade proximal do fêmur. Rev Bras Ortop. 2011;46(2):190-4.

11. Bento NT, Vidmar MF, Silveira MM, Wibelinger LM. Intervençôes fisioterapêuticas no pós-operatório de fratura de fêmur em idosos. Rev Bras Ciên Saúde. 2011;9(27):42-8.

12. Souza RC, Pinheiro RS, Coeli CM, Junior KRC, Torres TZG. Aplicaçáo de medidas de ajuste de risco para a mortalidade após fratura proximal de fêmur. Rev Saúde Pública. 2007;41(4):625-31.

13. Buckwalter JA. Ortopedia de Turek: princípios e sua aplicação. $5^{a}$ ed. São Paulo: Manole; 2000. 
14. Rocha MA, Carvalho WS, Zanqueta CL, Sandro C. Estudo epidemiológico retrospectivo das fraturas do fêmur proximal tratados no Hospital Escola da Faculdade de Medicina do Triângulo Mineiro. Rev Bras Ortop. 2001;36(8):311-6.

15. Moraes FB, Silva LL, Ferreira FV, Ferro AM, Rocha VL, Teixeira KS. Avaliação epidemiológica e radiológica das fraturas diafisárias do fêmur: estudo de 200 casos. Rev Bras Ortop. 2009;44(3):199-203.

16. Muniz CF, Arnaut AC, Yoshida M, Trelha CS. Caracterização dos idosos com fratura de fêmur proximal atendidos em hospital escola público. Rev Espaço Saúde. 2007;8(2):33-8.

17. Barbosa AD, Camargo CR, Arruda ES, Israel VL. Avaliaçáo fisioterapêutica aquática. Fisioterapia em Movimento. 2006;19(2):135-47.

18. Avelar NCP, BastoneAC,Alcântara MA, Gomes WF. Efetividade do treinamento de resistência à fadiga dos músculos dos membros inferiores dentro e fora d'água no equilíbrio estático e dinâmico de idosos. Rev Bras Fisioter. 2009;14(3):229-36.

19. Gomes MCSM. Comparação entre o tratamento no solo e na hidroterapia para pacientes com osteoporose: revisão da literatura. Rev Bras Ciên Saúde. 2006;7(3):45-56.

20. Candeloro JM, Caromano FA. Efeito de um programa de hidroterapia na flexibilidade e na força muscular de idosas. Rev Bras Fisioter. 2007;11(4):304-9.

21. Farias MA. Contribuição educativa e terapêutica da hidroterapia na qualidade de vida de pacientes portadores de osteoporose: análise de um programa de educação para a saúde. Rev Eletrônica do Mestrado Profissional em Ensino de Ciências da Saúde e do Ambiente. 2009;2(1):30-43.

22. Biasoli MC, Machado CMC. Hidroterapia: técnicas e aplicabilidades nas disfunçóes reumatológicas. Rev Temas de Reumatologia Clínica. 2006;7(3):78-87.

23. Caromano FA, Filho MRFT, Candeloro JM. Efeitos fisiológicos da imersão e do exercício na água. Rev Fisioterapia Brasil. 2003;4(1):1-5.

24. Hall CM, Brody LT. Exercício terapêutico: na busca da funçáo. Rio de Janeiro: Guanabara Koogan; 2001.

25. Wajchemberg M, Pires L, Rodrigues RC, Mano KS, Sottomaior MS, Cohen M, et al. Reabilitação precoce de atletas utilizando hidroterapia após o tratamento cirúrgico de hérnia discal lombar: relato preliminar de 3 casos. Acta Ortop Bras. 2002;10(2):48-57.

26. Resende SM, Rassi CM, Viana FP. Effects of hydrotherapy in balance and prevention of falls among elderly women. Rev Bras Fisiot. 2008;12(1):57-63.

27. Lustosa LP, Bastos EO. Fraturas proximais do fêmur em idosos: qual o melhor tratamento? Acta Ortop Bras. 2009;17(5):309-12.

28. Pesciallo C, Pastrián Mana D, Barrios JM, Del Sel H. Fracturas subtrocantéreas de fémur: Tratamiento con clavo de fémur proximal por técnica mínimamente invasiva. Rev Asoc Argent Ortop Traumatol. 2009;74(1):13-9.

29. Guimarães JAM, Guimarães ACA, Franco JS. Avaliação do emprego da haste femoral curta na fratura trocantérica instável do fêmur. Rev Bras Ortop. 2008;43(9):406-17.

30. Murphy S, Conway C, McGrath NB, O'Leary B, O’Sullivan MP, O'Sullivan D. An intervention study exploring the effects of providing older adult hip fracture patients with an information booklet in the early postoperative period. J Clin Nursing. 2011;20(23-24):3404-13.

31. Meereis ECW, Favretto C, Souza J, Marques CLS, Gonçalves MP, Mota CB. Análise do equilíbrio dinâmico de idosas institucionalizadas após hidrocinesioterapia. Rev Bras Geriatr Gerontol. 2013;16(1):41-7.

32. Katrak P, O'Connor B, Woodgate I. Rehabilitation after total femur replacement: a report of 2 cases. Arch Phys Med Rehabil. 2003;84(7):1080-4.

33. Campion MR. Hidroterapia: princípios e prática. São Paulo: Manole; 2000.

34. Ruoti RG, Morris DM, Cole AJ. Reabilitação aquática. São Paulo: Manole; 2000.

35. Maia FES, Almeida JRS, Queiroz JM, Mendes JMR, Lima e Silva J. Comissão interna de prevenção de acidentes e as açóes de saúde coletiva na perspectiva da fisioterapia. Rev Fisioter Saúde Fun. 2014;3(2):6-12.

36. Silva EL, Menezes EM. Metodologia da pesquisa e elaboraçâo de dissertação. $4^{\mathrm{a}}$ ed. rev. atual. [Internet] Florianópolis: UFSC; 2005 [acesso em 21 dez 2013]. Disponível em: $\mathrm{ftp}$ //ftp.unilins.edu.br/brenoortega/metodologia/metodologia_de_pesquisa.pdf

37. Silva MV, Santiago RF, Coelho DMM. Utilizaçáo da hidroterapia como prática alternativa: uma revisão de literatura. In: 61 ${ }^{\circ}$ Congresso Brasileiro de Enfermagem. Transformação social e sustentabilidade ambiental. Trabalho 2652-1/5. Fortaleza/CE. 2009 [acesso em 18 dez 2013], p. 6848-52. Disponível em: http://www.abeneventos.com.br/ anais_61cben/files/02074.pdf

38. Miotto C, Kayser B, Molin VD, Kummer JÁ, Wibelinger LM. Tratamento fisioterapêutico das artralgias. Rev Dor. 2013;14(3):216-8.

39. Silva DM, Nunes MCO, Oliveira PJAL, Coriolano MGWS, Berenguer FA, Lins OG, et al. Efeitos da fisioterapia aquática na qualidade de vida de sujeitos com doença de Parkinson. Fisioter Pesqui. 2013;20(1):17-23.

40. Caldart J, Faggion M, Santos RV. Eficácia da hidrocinesioterapia na fratura de joelho: estudo de caso. Rev FisiSenectus. 2013;(1):63-9.

41. Rosa TS, Andriollo DB, Vey APZ, Mai CG. Fisioterapia aquática como prevenção de quedas na terceira idade: revisão de literatura. Cinergis. 2013;14(1):25-8.

42. Almeida CI, Saad M, Vieira MSR. Reabilitação em reumatologia geriátrica. Einstein. 2008;6(Supl 1):183-9.

43. Bruni BM, Granado FB, Prado RA. Avaliação do equilíbrio postural em idosos praticantes de hidroterapia em grupo. Mundo Saúde. 2008;32(1):56-63.

44. Granillo ZM, Castillo CIRÁ, Suárez JMR, Pérez JAA. Tratamiento de neuroterapia acuática en niños menores de 4 años. Plasticidad y Restauración Neurológica. 2006;5(1):25-9. 
45. Teixeira CS, Pereira EF, Rossi AG. A hidroginástica como meio para manutençáo da qualidade de vida e saúde do idoso. Acta fisiátrica. 2007;14(4):226-32.

46. Wajchemberg M, Pires L, Rodrigues RC, Mano KS, Sottomaior MS, Cohen M, et al. Reabilitação precoce de atletas utilizando hidroterapia após o tratamento cirúrgico de hérnia discal lombar: relato preliminar de 3 casos. Acta ortop bras. 2002;10(2):48-57.

47. Jacques KC, Drumond NR, Andrade SAF, Júnior IPC, Toffol WC. Eficácia da hidroterapia em crianças com encefalopatia crônica não progressiva da infância: revisão sistemática. Fisioter Mov. 2010;23(1):53-61.

48. Hay L. Best BETs from the Manchester Royal Infirmary. BET 3: does hydrotherapy help improve post ankle fracture symptoms? Emerg Med J. 2013;30(9):771.

49. Langschmidt J, Caine PL, Wearn CM, Bamford A, Wilson YT, Moiemen NS. Hydrotherapy in burn care: a survey of hydrotherapy practices in the UK and Ireland and literature review. Burns. 2013;40(5):860-4.

50. Ramos JM, Mansoldo AC. Efeito de 8 meses de hidroginástica em idosas com osteoporose. Motriz rev educ fis. 2007;13(2):123-8.

51. Becker BE, Cole AJ. Terapia aquática moderna. São Paulo: Manole; 2000.

52. Silva WM, Martin DG. Proposta de protocolo hidrocinesioterapêutico para tratamento de luxação congênita de quadril, um estudo de caso. XI Salão de Iniciação Científica PUCRS, 9 a 12 de agosto de 2010 [acesso em 18 dez 2013], p. 502-4. Disponível em: http://www.pucrs.br/edipucrs/
XISalaoIC/Ciencias_da_Saude/Fisioterapia_e_Terapia_ Ocupacional/83362-WILLIAM_MACEDO_SILVA.pdf

53. Handoll HHG, Sherrington C. Mobilisation strategies after hip fracture surgery in adults. Cochrane Database Syst Rev. 2007;24(1):CD001704.

54. Ferreira A, Almeida D, Campos W, Campos F, Tomazelli R, Romão D. Incidência e caracterização de idosos na clínica ortopédica do Hospital Regional Cáceres por fratura de fêmur. Rev Eletrônica Gestão \& Saúde. 2013;4(2):52-62.

55. Brech GC, Guarniero R, Junior RMG. Amplitude de movimento articular do quadril em pacientes com a doença de Legg-Calvé-Perthes. Fisioter Mov. 2008;21(1):131-8.

56. Paião RCN, Dias LIN. A atuação da fisioterapia nos cuidados paliativos da criança com câncer. Ensaios e Ciência: C. Biológicas, Agrárias e da Saúde. 2012;16(4):153-69.

57. Vidmar MF, Muntini N, Audino LP, Almeida CR, Pimentel GL. Efeito da mobilização passiva contínua em pós-operatório de lesão condral traumática do joelho: revisão de literatura. Rev Ciên Méd Biol. 2013;12(2):237-42.

58. Moller R. Terapias holísticas no esporte. Sáo Paulo: IBRASA; 2000.

59. Chaskel CF, Preis C, Neto LB. Propriocepção na prevenção e tratamento de lesôes nos esportes. Rev Ciên Saúde. 2013;6(1):67-76.

60. Maia FES, Medeiros EC, Carvalho RRP, Pinto AGKO, Moura ELR, Silva SAL, et al. O alongamento estático manual da musculatura envolvida na inspiração pode promover benefícios para os volumes inspiratórios? Rev Ter Manual. 2013;11(53):415-20.

\section{Como citar este artigo:}

Maia FES, Gurgel FFA. Existe fundamento científico para o tratamento da fratura de fêmur pela hidroterapia? Uma revisão integrativa. Rev. Aten. Saúde. 2016;14(49):104-110. 